\title{
Tumor-penetrating peptide fused to a pro-apoptotic peptide facilitates effective gastric cancer therapy
}

\author{
YING HUANG $^{1 *}$, XIHAN LI $^{2}$, HUIZI SHA ${ }^{3}$, LIANRU ZHANG $^{3}$, \\ XINYU BIAN ${ }^{3}$, XIAO HAN ${ }^{4}$ and BAORUI LIU ${ }^{1}$ \\ ${ }^{1}$ The Comprehensive Cancer Centre, Nanjing Drum Tower Hospital Clinical College of Nanjing Medical University; \\ ${ }^{2}$ State Key Laboratory of Pharmaceutical Biotechnology, School of Life Sciences, Nanjing University; \\ ${ }^{3}$ The Comprehensive Cancer Centre of Drum Tower Hospital, Medical School of Nanjing University \\ and Clinical Cancer Institute of Nanjing University; ${ }^{4}$ Key Laboratory of Human Functional Genomics \\ of Jiangsu Province, Jiangsu Diabetes Center, Nanjing Medical University, Nanjing, Jiangsu, P.R. China
}

Received July 14, 2016; Accepted September 6, 2016

DOI: $10.3892 /$ or.2017.5440

\begin{abstract}
KLA (sequence, KLAKLAKKLAKLAK) is a peptide which leads to programmed cell death by disrupting the mitochondrial membrane. However, low penetration in tumors greatly limits its application and efficacy. To develop a KLA-based cancer therapy, KLA-iRGD, a recombinant protein was constructed. It consists of the KLA peptide and iRGD (CRGDKGPDC), a tumor-homing peptide with high penetration into tumor tissue and cells. The conjugated KLA exhibits pro-apoptotic activity to prevent the growth of a tumor once it is inside the cell. Once KLA-iRGD is internalized in cultured tumor cells, via the activation of the receptor neuropilin-1, it spreads extensively throughout the mass of the tumor. The recombinant KLA-iRGD protein showed antitumor activity in vivo in mice and in vitro in tumor cell lines. Repeated treatment with KLA-iRGD greatly prevented tumor growth, resulting in a considerable reduction in tumor volume. According to our data, KLA-iRGD may serve as a potential anticancer agent with limited systemic toxicity and high selectivity for the treatment of MKN45 gastric cancer, which may lead to the enhancement of new targeted anticancer agents.
\end{abstract}

Correspondence to: Dr Baorui Liu, The Comprehensive Cancer Centre, Gulou Clinical Medical School of Nanjing Medical University, Nanjing, Jiangsu, P.R. China

E-mail: baoruiliu@nju.edu.cn

Dr Xiao Han, Key Laboratory of Human Functional Genomics of Jiangsu Province, Jiangsu Diabetes Center, Nanjing Medical University, Nanjing, Jiangsu, P.R. China

E-mail: hanxiao@njmu.edu.cn

${ }^{*}$ Contributed equally

Key words: recombinant protein, iRGD, KLA, drug penetration

\section{Introduction}

As a leading cause of cancer-related death, gastric cancer has a high rate of mortality and incidence, particularly in the Eastern world $(1,2)$. Despite the recent progress in cancer therapy, including biological immune therapy, radiotherapy and chemotherapy, a majority of malignancies are still not curable today (3). Therefore, it is important to conduct studies to discover and develop advanced therapeutics.

Programmed cell death has been considered as a defense mechanism whereby defective and harmful cells are eliminated (4). Disruption of programmed cell death signaling pathways may result in elevated cell proliferation and eventually cancer. As a cationic amphipathic peptide which disrupts the mitochondrial membrane, KLA (sequence, KLAKLAKKLAKLAK) may lead to mitochondrial swelling and permeabilization (5-7), and ultimately programmed cell death by causing the release of cytochrome $c$ (8-10). However, this peptide fails to effectively permeate the eukaryotic plasma membrane and in the end shows low cytotoxicity in mammalian cells $(11,12)$. Accordingly, KLA requires the assistance of other cell penetrating peptides for effective translocation in micromolar concentrations. As KLA can pass through the plasma membrane, it preferentially interferes with the charged mitochondrial membrane to release cytochrome $c$, causes disruption in mitochondrial membrane potential and eventually induces cell death $(13,14)$.

We undertook the present study to fully understand the action mechanism of KLA, and explore its use as an antitumor drug. In the present study, to circumvent the difficulties of low drug penetration into solid tumors and to efficiently deliver KLA into the tumor, CRGDKGPDC, a C-end rule peptide iRGD, was introduced into KLA. Similar to regular peptides of the RGD (Arg-Asp-Gly) type, iRGD mediates tumorhoming by first binding to $\alpha \mathrm{v}$ integrins, which are selectively expressed in various cell types in tumors and the endothelium of tumor vessels, but not in normal tissues (15). When proteolytically cleaved in tumors, iRGD generates CRGDK/R which has an affinity for NRP-1, a receptor which becomes activated 
Table I. Peptide sequences and molecular weight of the peptides.

\begin{tabular}{lcccc}
\hline \multirow{2}{*}{ Peptides } & \multicolumn{3}{c}{ Sequences } & \\
\cline { 2 - 4 } & Protein transduction domain & Linker & Pro-apoptotic domain & Expected molecular weight (Da) \\
\hline KLA & & & KLAKLAKKLAKLAK & $1,524.01$ \\
KLA-iRGD & CRGDKGPDC & \multirow{2}{*}{ GGGGS GGGGS } & KLAKLAKKLAKLAK & $3,086.62$ \\
KLA-RGD & ACDCRGDCFC & GGGGS GGGGS & KLAKLAKKLAKLAK & $3,228.81$ \\
\hline
\end{tabular}

and mediates its penetration into tissue (16). KLA-iRGD was shown to exhibit all the characteristics of a tumor-specific homing peptide with tissue penetration activity. Additionally, the penetration of KLA-iRGD was assessed using in vivo approaches. Finally, the anticancer activity of the KLA-iRGD recombinant protein was examined.

\section{Materials and methods}

Reagents, cell lines and tumors. Fluorescein isothiocyanate (FITC) was obtained from Sigma Chemical Co. (St. Louis, MO, USA). DAPI was purchased from Beyotime Biotechnology (Wuhan, China). To assay for cell viability, 3-(4,5-dimethylthiazol-2-yl)-2,5-diphenyltetrazolium bromide (MTT) was obtained from Sigma Chemical Co. The Annexin V-FLUOS staining kit was purchased from Roche Applied Science (Indianapolis, IN, USA). The primary antibodies mouse monoclonal anti-human CD31 (GM082301 $1 \mathrm{ml}$ RTU) were purchased from Gene-Tech Co. Ltd. (Shanghai, China). The secondary antibody rat monoclonal anti-mouse CD31 (553370; $0.5 \mathrm{mg} / \mathrm{ml}$ ) was purchased from BD Pharmingen (San Jose, CA, USA). The Cell Death Detection kit for terminal deoxynucleotidyl transferase-mediated dUTP nick end labeling (TUNEL) was acquired from Roche Applied Science. The $\alpha v \beta 3$ antibody was purchased from Cell Signaling Technology (Danvers, MA, USA). The human NRP-1 antibody was purchased from Abcam (Cambridge, MA, USA).

The human gastric carcinoma cell lines MKN45 and KATO III were purchased from the Cell Bank of the Shanghai Institute of Biochemistry and Cell Biology (Shanghai, China). All human cell lines were cultured in RPMI-1640 medium with $10 \%$ fetal bovine serum (FBS) (both from Invitrogen, Carlsbad, CA, USA), with glutamine, streptomycin and penicillin at $37^{\circ} \mathrm{C}$ and $5 \% \mathrm{CO}_{2}$.

$\mathrm{BALB} / \mathrm{c}$ mice (male, 18-22 $\mathrm{g}$ and 5- to 6-weeks old) were purchased from the BK Lab Animal Ltd. (Shanghai, China), and were maintained at $25 \pm 1^{\circ} \mathrm{C}$, under specific pathogen-free (SPF) condition, and were given access to food and water ad libitum. All animal procedures were conducted according to the regulations of the Animal Care Committee at Drum Tower Hospital (Nanjing, China). MKN45 gastric cancer cells $\left(5 \times 10^{6}\right)$ in $0.1 \mathrm{ml}$ phosphate-buffered saline (PBS) were subcutaneously injected into the lower right flank of athymic nude BALB/c mice, so as to generate tumor xenografts. The volume of the tumors was computed with a digital Vernier caliper and using the following formula: Tumor volume $=\left(\right.$ length $\mathrm{x}$ width $\left.{ }^{2}\right) / 2$, where the width has the widest dimension and the length has the longest dimension.
Peptide synthesis. High purity ( $>90 \%$ ) peptides were obtained from Bankpeptide Biological Technology Co. Ltd. (Hefei, China). An integrated product of iRGD and KLA was generated for delivery into the mitochondria. Two unstructured glycine residues, as a spacer, were inserted. All the peptide products were analyzed by analytic high-pressure liquid chromatography. Additionally, the predicted molecular weight (MW) of the resulting conjugates are listed in Table I. Freeze-dried peptides were reconstituted in high-purity dimethyl sulfoxide (DMSO) $(10 \mathrm{mM})$ and kept at $-70^{\circ} \mathrm{C}$ until use.

Western blotting to assess $\alpha v \beta 3$ and NRP-1 expression. Protein extracts were prepared from MKN45 and KATO III cells $\left(2 \times 10^{6}\right)$, cultured for $30 \mathrm{~min}$, using the following protein lysis buffer: $150 \mathrm{mM} \mathrm{NaCl}, 1 \mathrm{mM}$ dithiothreitol (Merck, Cefoperazone, Germany), $50 \mathrm{mM}$ Tris- $\mathrm{HCl}$ ( $\mathrm{pH} 7.5), 0.1 \%$ sodium dodecyl sulfate (SDS), and protease inhibitors (Roche, Basel, Switzerland). The extracts were separated using SDS polyacrylamide gel electrophoresis and electrophoretically transferred to nitrocellulose membrane (Millipore, Waltham, MA, USA). Standard western blot analyses were conducted using the antibodies against $\alpha v \beta 3$ and NRP-1. The blots were incubated at room temperature for $1 \mathrm{~h}$ with the antibody conjugated to horseradish peroxidase (HRP) and detected with an enhanced chemiluminescence reagent (Cell Signaling Technology Inc., USA). The autoradiographic intensity of each band was scanned and quantified using BandScan software (Glyko, Inc., Novato, CA, USA). Values were normalized to GAPDH and the ratio to the control values was calculated.

Cell internalization of the recombinant proteins. MKN45 and KATO III cells, in the logarithmic growth phase, were seeded at $1.0 \times 10^{6} /$ well in 24 -well chamber slides (Nalge Nunc International Corp., Rochester, NY, USA). Subconfluent tumor cells on the chamber slides were incubated for $1 \mathrm{~h}$ with $10 \mu \mathrm{g} / \mathrm{ml}$ FITC-labelled protein. Afterwards, the cells were rinsed 3 times with PBS ( $\mathrm{pH} 7.4)$ and fixed with ice-cold methanol for $10 \mathrm{~min}$. The cells were then rinsed 3 times with PBS ( $\mathrm{pH} 7.4)$ and the nuclei were stained with DAPI. Subsequently, the cells were observed with a laser scanning confocal microscope (LSCM; Zeiss LSM 710).

Antiproliferation assay. MKN45 and KATO III cells, in the logarithmic growth phase, were seeded at $5 \times 10^{3}$ cells/well in 96-well plates and cultured in a humid environment at $37^{\circ} \mathrm{C}$ in $5 \% \mathrm{CO}_{2}$ for $12 \mathrm{~h}$. Thereafter, the medium was removed, and the cells were exposed to serum-free medium containing KLA, KLA-iRGD and KLA-RGD. After incubation for $24 \mathrm{~h}$, 
the viability of the cells was evaluated using an MTT assay following the manufacturer's instructions. The cell viability values were calculated by a nonlinear regression study utilizing GraphPad Prism v5.0 software (San Diego, CA, USA).

Flow cytometric analysis. The induction of apoptosis was detected with the Annexin V-FLUOS staining kit following the manufacturer's instructions. Briefly, the cells were cultured at a density of $1.0 \times 10^{6}$ cells $/ \mathrm{ml}$ at $37^{\circ} \mathrm{C}$ in $5 \% \mathrm{CO}_{2}$, treated with $100 \mathrm{ng} / \mathrm{ml}$ fusion protein (KLA, KLA-iRGD and KLA-RGD), and continued to culture for $4 \mathrm{~h}$. Afterwards, the cells were washed 2 times with cold PBS and a volume of $100 \mu 1$ of Annexin V-FLUOS was added to the cells. Then, all cell samples were cultured in the dark for $15 \mathrm{~min}$ at room temperature and gently vortexed, before flow cytometric analysis on a FACSort flow cytometer (Becton-Dickinson, San Jose, CA, USA).

Tumor tissue penetration in vivo. An animal model was used to study the recombinant protein penetration and to trace the location of the protein. BALB/c mice were subcutaneously inoculated with MKN45 cells. The MKN45 tumor-bearing mice were inoculated via a tail vein with FITC-labeled KLA-iRGD when tumors of $\sim 200 \mathrm{~mm}^{3}$ were formed. One hour after administration of the peptide, the mice were sacrificed and tumors were harvested. As mentioned previously, immunofluorescence analysis was carried out with frozen tumor tissues. For immunostaining, the slides were first blocked at room temperature with $20 \%$ goat serum for $1 \mathrm{~h}$, and then incubated with an anti-mouse antibody against CD31 (1x300 dilution) at $4^{\circ} \mathrm{C}$ overnight. Finally, the slides were subjected to DAPI staining for nuclear counterstaining and visualized with a LSCM laser scanning confocal microscope.

Tumor treatment in nude mice. Animal handling was performed in accordance with the protocols of the Institutional Animal Care and Use Committee (IACUC) of Ohio State University. Five-week-old BALB/c nude mice were subcutaneously implanted with $2 \times 10^{6}$ MKN45 cells in the right flank. Tumor-bearing mice were assigned to 3 treatment groups $\sim 2$ weeks after the inoculation of the MKN45 cells. The assignment was based on tumor size to ensure that there was no statistical difference in tumor volume among the groups when the treatment began. The peptide molecules, at $1 \mathrm{mg} /$ $\mathrm{ml}$ in PBS, were intraperitoneally injected. A total of 4 injections were administered into the mice of the MKN45 model every 3 days. The volume of the tumor was evaluated with an electronic caliper and the tumor volume was computed from measurements of 2 parameters utilizing the following formula: Tumor volume $=\left(\right.$ length $\mathrm{x}$ width $\left.^{2}\right) / 2$. Based on the guideline of IACUC, the experiments were halted when the diameter of the tumors reached $1.5 \mathrm{~cm}$. Finally, xenografts were weighed and removed and mice were sacrificed. Regarding the toxicological assessment of the peptide treatments, organs, including the heart, kidney, liver, spleen and lung, were fixed in neutral buffered formalin, embedded in paraffin, and stained with hematoxylin and eosin (H\&E) for pathological study after the animals were euthanized. TUNEL assay was used for evaluation of tumor cell apoptosis. The collected slices were evaluated using optical microscopy.
Statistical analysis. All the in vitro experiments were repeated at least 3 times. Statistical analysis was conducted using GraphPad Prism version 5.0 (GraphPad Software). Statistical values are expressed as means $\pm \mathrm{SD}$. The statistical analysis was performed using the two-tailed Student's t-test, followed by the Bonferroni test. Different group comparison was conducted by one-way analysis of variance (ANOVA). Two-way ANOVA was used to examine whether the difference between the data from the various groups was significantly different regarding peptide and the effects of the fused peptides for every parameter. A p-value of $<0.05$ was regarded as significantly different.

\section{Results}

Internalization of the recombinant proteins into cells. To deliver the KLA molecule into cells and potentially into the cytoplasm, we fused KLA to a tumor-penetrating iRGD peptide. For comparison, we also used RGD (sequence: ACDCRGDCFC), a peptide in which the CendR motif is constitutively active. The internalization of the conjugate by MKN45 and KATO III cells was studied by LSCM using FITC-labeled KLA, KLA-iRGD and KLA-RGD. KLA with no CendR peptide showed negligible binding to the MKN45 cells (Fig. 1A), a cell line that expresses high levels of NRP-1 and $\alpha v \beta 3$ integrin (Fig. 1B and C). Additionally, KLA also showed negligible binding to the KATO III cells, a cell line that expresses low levels of NRP-1 and $\alpha v \beta 3$ integrin. In contrast, both KLA-iRGD and KLA-RGD effectively bound to the MKN45 cells, but KLA-RGD was only weakly internalized. As expected, KLA-iRGD and KLA-RGD did not bind to or were internalized into the NRP-1-deficient KATO III cells. The entry of KLA-iRGD into the cells was rapid, as indicated by the finding that after a 30 -min incubation, the protein was detectable in the MKN45 cells.

Reduction in cell viability by recombinant proteins. In order to confirm the pharmacological activity of KLA-iRGD, in vitro cytotoxicity analyses were performed using the MTT assay to monitor the viability of the MKM45 and KATO III cells. The results revealed that KLA-RGD had no antiproliferative activity against the MKN45 cells at the highest concentration $(200 \mu \mathrm{g} / \mathrm{ml})$ tested compared with KLA ( $\mathrm{p}=0.136)$ (Fig. 2A). In contrast, a dose-dependent cytotoxicity was detected for KLA-iRGD ( $p=0.002)$, indicating that the extra iRGD motif enhanced the antiproliferative activity of KLA-iRGD in the human gastric cancer cell line MKN45. Regarding the cell line KATO III (Fig. 2B), KLA-iRGD and KLA-RGD exhibited no obvious antiproliferative activity compared with KLA $(\mathrm{p}=0.144$ and $\mathrm{p}=0.287)$. Although KLA-iRGD exhibited a stronger inhibitory effect on KATO III proliferation, the differences between KLA, KLA-iRGD and KLA-RGD were not evident.

To ascertain whether these fusion peptides induce apoptosis, we analyzed the apoptotic rates by flow cytometric analysis. The overlapping effects in the MKN45 cells were measured by an Annexin V-FLUOS staining apoptosis assay. The finding of differential staining can be expressed as the percentage of positive cells compared to the total number of cells. Our results revealed that both KLA and KLA-RGD induced low levels of apoptosis in the MKN45 cells (Fig. 3A), 
A
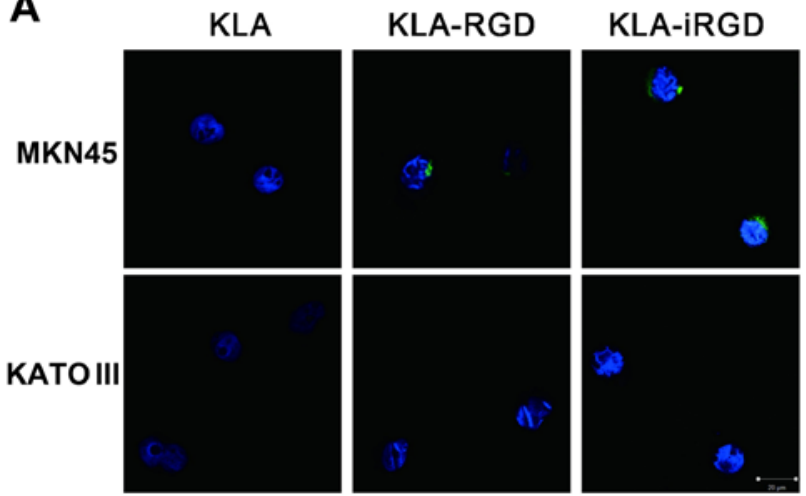

B

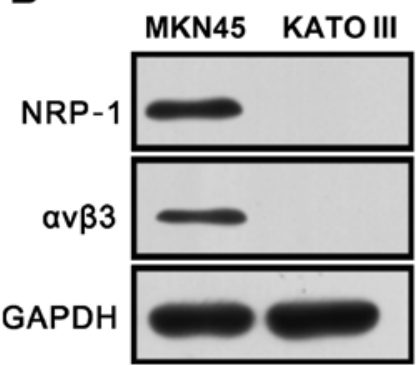

C

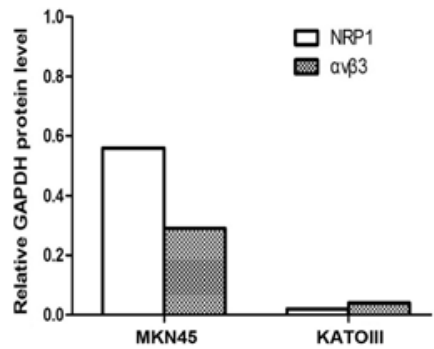

Figure 1. (A) Internalization of KLA, KLA-iRGD and KLA-RGD into the MKN45 and KATO III cells. The MKN45 cells, which are positive for NRP-1 and $\alpha v \beta 3$ integrin, and NRP-1-deficient, $\alpha v \beta 3$-deficient KATO III cells were incubated with $10 \mu \mathrm{g} / \mathrm{ml}$ of FITC-labeled fusion proteins (green). The cells were treated with methanol for $10 \mathrm{~min}$, and then washed with PBS. DAPI (blue) was used to stain the nuclei. The proteins were visualized by confocal microscopy. MKN45 and KATO III cells were incubated with KLA, KLA-iRGD and KLA-RGD for $30 \mathrm{~min}$. Scale bar, $20 \mu \mathrm{m}$. (B) Evaluation of $\alpha v \beta 3$ and NRP-1 protein leveld by western blot analysis in yjr MKN45 and KATOIII celld. (C) Quantification of protein expression.

as indicated by the percentages of early and late apoptotic MKN45 cells following treatment with KLA and KLA-RGD $(4.3 \pm 2.2$ and $11.9 \pm 3.9 \%$, respectively; $p=0.115)$. In comparison, KLA-iRGD induced not only apoptosis, but also necrosis as shown in Fig. 3A, and the percentage of early and late apoptosis was much higher $(63.9 \pm 17.0 \%)$ compared with KLA and KLA-RGD ( $p=0.023$ and $p=0.024)$. According to these findings, KLA-iRGD increased apoptosis in vitro and effectively entered into cancer cells (Fig. 3B).

Penetration of KLA-iRGD into tumor tissue. Through the use of tumor tissue derived from the MKN45 tumor-bearing mice, we studied the penetration capability of KLA-iRGD (Fig. 4A). According to the fluorescence signal on the LSCM images, the locations of the recombinant protein were displayed as FITClabelled KLA, KLA-iRGD and KLA-RGD. An anti-CD31 antibody and a Cy3-conjugated secondary antibody bound to blood vessels. Therefore, red fluorescence was detected in blood vessels. Actually, KLA-RGD was localized in the vicinity of blood vessels $1 \mathrm{~h}$ post-injection, and it was additionally found that it could be targeted to the tumor tissue. In contrast, the KLA-iRGD peptide was found to penetrate into extravascular tumor tissue, with a tumor penetration activity that was readily apparent. In fact, a strong KLA-iRGD signal was detectable in all sections from the entire tumor. KLA-iRGD traveled further from the tumorous vessels and was found on a greater field in
A

\section{MKN45}

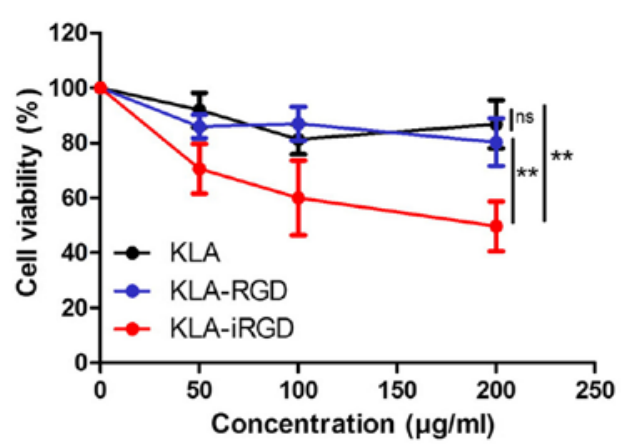

B

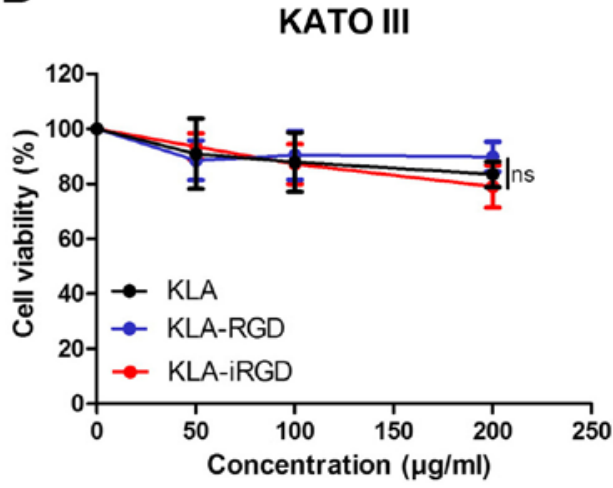

Figure 2. Cytotoxicity of the protein KLA-iRGD in tumor cell lines. KLA, KLA-iRGD and KLA-RGD were added, at various concentrations, to the (A) MKN45 and (B) KATO III cell suspensions and incubated for $48 \mathrm{~h}$. Cell viability was measured by the MTT assay. All data are expressed as the mean \pm standard deviation $(\mathrm{SD}), \mathrm{n}=3$. Data represent the means of 3 independent experiments. Statistical analyses were performed using the Student's t-test and one-way ANOVA; ${ }^{* *} \mathrm{p}<0.01$; ns, not significant.

the tumor tissues in comparison with KLA-RGD. Specifically, the FITC fluorescence mean intensity of KLA-RGD was 5 times weaker than that of KLA-iRGD (Fig. 4B). All the statistical analysis results support the notion that as a functional group, iRGD mediates the active tumor penetration of KLA-iRGD protein.

Inhibitory effect of KLA-iRGD in the xenograft model. In order to examine the in vivo antitumor effects of KLA-iRGD, we systemically administered the peptide into nude mice with established MKN45 tumors. We initially injected KLA and KLA-iRGD intraperitoneally at $10 \mathrm{mg} / \mathrm{kg}$ of mouse body weight every 3 days. The tumor size was measured during the different treatment times. The size of the MKN45 tumors increased rapidly in mice intraperitoneally injected with PBS. In comparison, in the KLA-iRGD-treated mice, the inhibition of tumor growth was evident as early as 3 days after treatment. Representative images of nude mice with established MKN45 tumors after a 12-day post treatment period with KLA-iRGD, KLA or PBS as control are presented in Fig. 5A. The timedependent changes in MKN45 tumor growth in the nude mice are shown in Fig. 5B. Clearly, systemic delivery of KLA-iRGD led to the effective suppression of MKN45 tumor growth in the xenograft model. After completion of the xenograft experiment, we dissected and weighed the MKN45 tumor xenografts. 


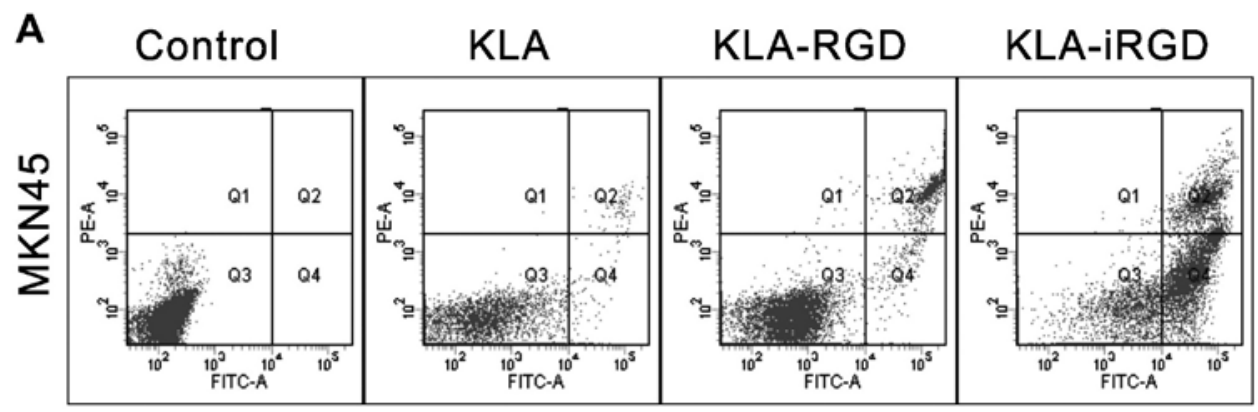

B

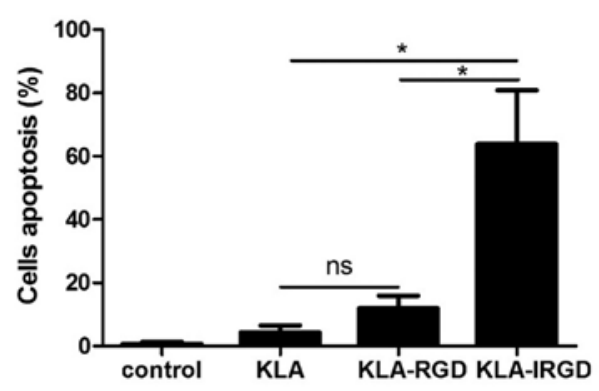

Figure 3. KLA-iRGD induces tumor cell apoptosis in vitro. (A) Cell apoptosis in the MKN45 cells after the addition of $100 \mathrm{ng} / \mathrm{ml}$ KLA, KLA-RGD and KLA-iRGD were assessed using the Annexin V/PI apoptosis assay. (B) Percentages of apoptotic cells as detected by flow cytometry. The average percentage of apoptotic cells $( \pm \mathrm{SD})$ is shown; ${ }^{*} \mathrm{p}<0.05$; ns, not significant.

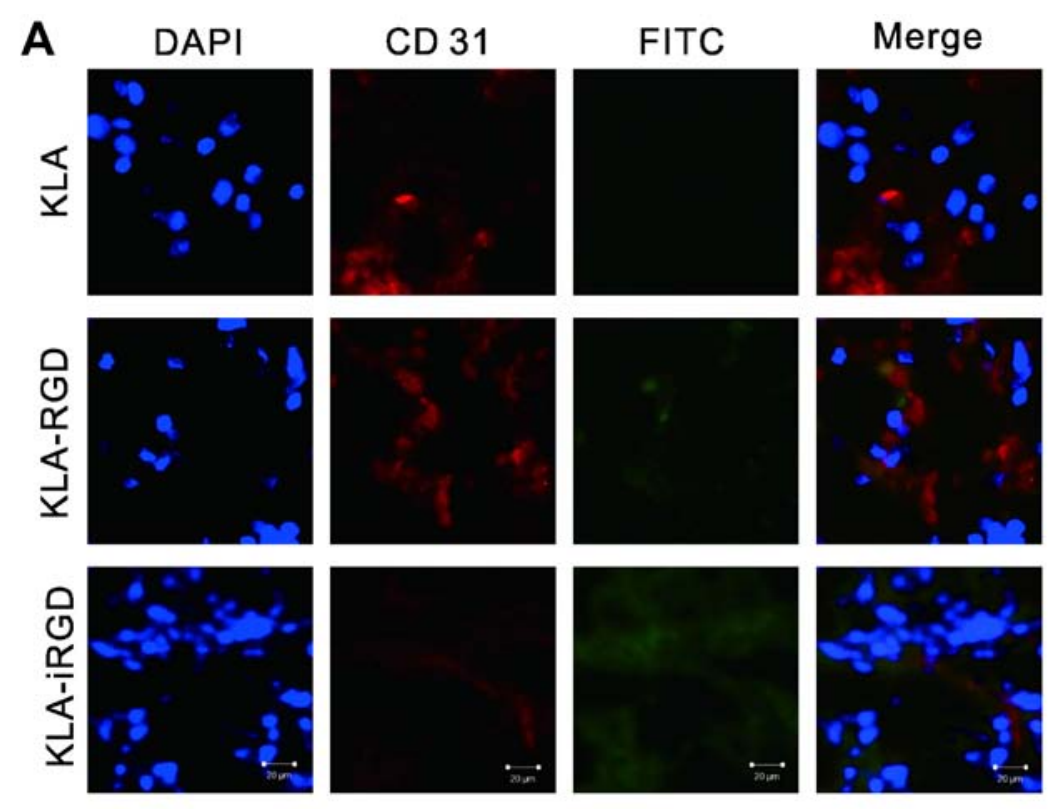

B

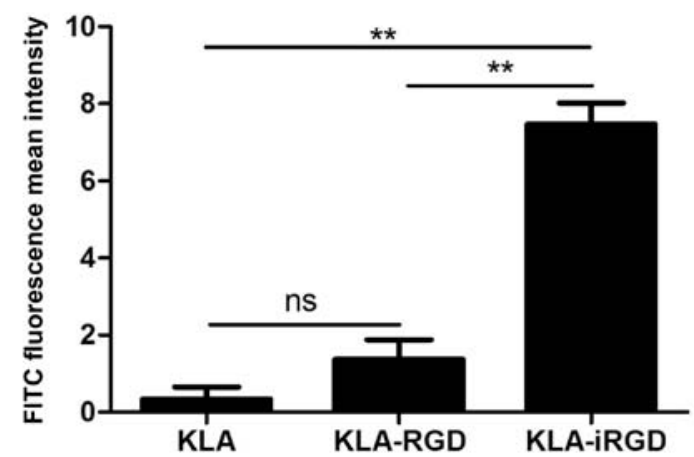

Figure 4. Tumor penetration of the KLA, KLA-iRGD and KLA-RGD peptides into the MKN45 tumor-bearing mice at $1 \mathrm{~h}$ post-injection. (A) Frozen tissue sections were examined under a confocal microscope. Blood vessels were visualized with anti-CD31 (red), nuclei were stained with DAPI (blue), while the protein peptides were labeled in green. Scale bar, $20 \mu \mathrm{m}$. (B) Quantification of the fluorescence intensity. Error bars denote mean \pm SD; * $p<0.01$; ns, not significant. 
A
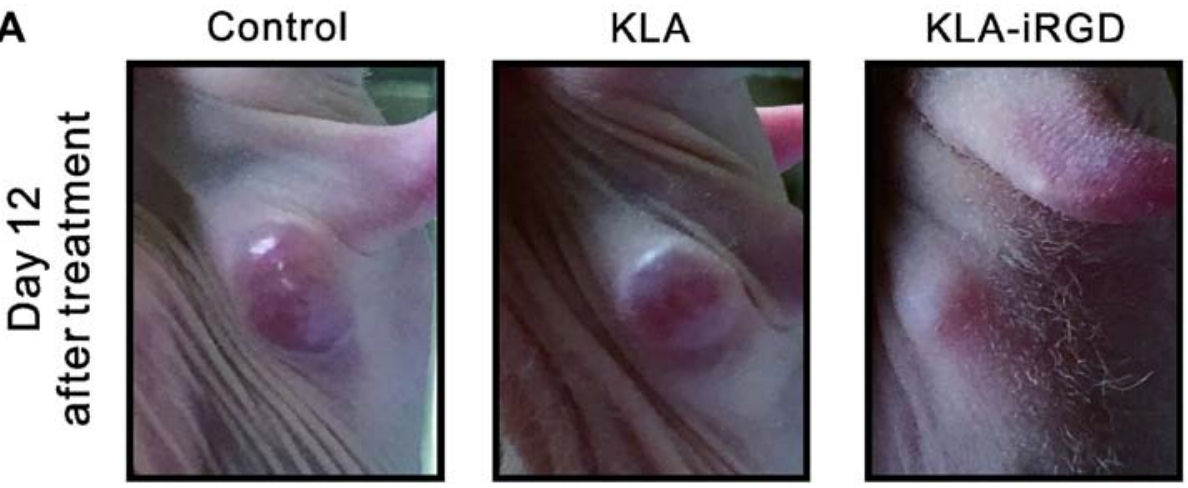

B
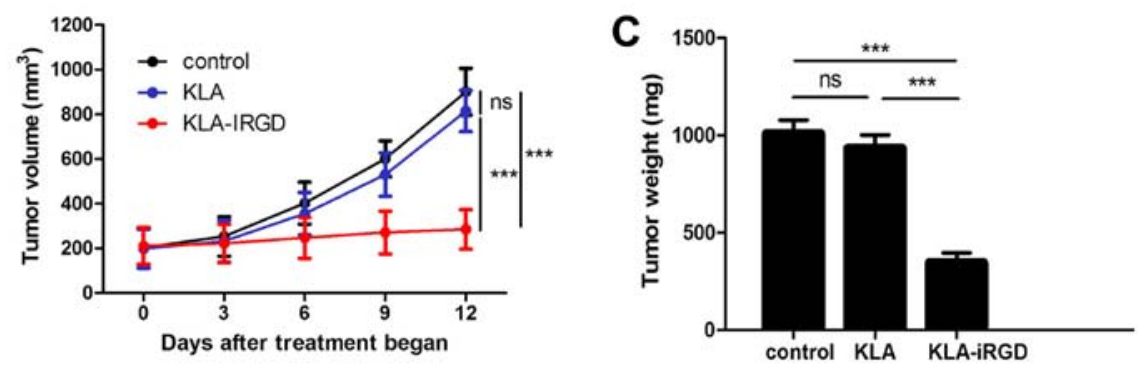

D

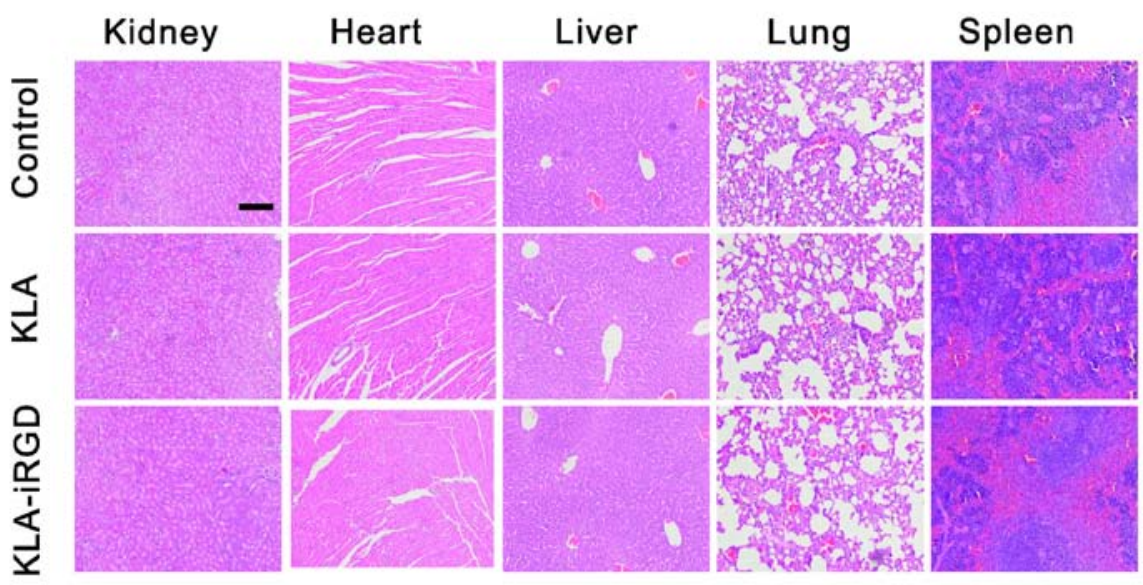

Figure 5. KLA-iRGD suppresses tumor growth with no toxic effects in the xenograft model. (A) MKN45 cells were subcutaneously injected into the right flanks of nude mice and xenografts were allowed to grow for 2 weeks. KLA-iRGD $(10 \mathrm{mg} / \mathrm{kg})$, KLA or PBS was administered into the mice via intraperitoneal injection. Images were captured 12 days after treatment. (B) The MKN45 xenograft volume from animals treated with KLA-iRGD was significantly smaller than the tumor volumes observed in the animals treated with the control KLA or PBS $(n=6)$. (C) The mean tumor weight showed significant suppression following treatment with KLA-iRGD. (D) Morphological details were examined using H\&E staining. No significant pathological changes were observed in the kidney, heart, liver, lung and spleen of nude mice following treatment with KLA-iRGD. Scale bar, $50 \mu \mathrm{m}$. Two-way ANOVA was used for the analysis of tumor volume and one-way ANOVA for the tumor weight; ${ }^{* * *} \mathrm{p}<0.001 ; \mathrm{ns}$, not significant.

The results shown in Fig. 5C, revealed that KLA-iRGD significantly suppressed MKN45 tumor growth. To test the potential toxicity of KLA-iRGD in the experimental mice, H\&E-stained section of the kidney, lung, heart, liver and spleen were examined after peptide treatment. As shown in Fig. 5D, there were no obvious changes in these organs following treatment with KLA-iRGD compared with the control. Additionally, no skin abnormality around the injection sites was observed, suggesting the tumor-specific penetration of KLA-iRGD.

H\&E-stained tumor sections showed differences among tumors treated with KLA-iRGD, KLA or PBS (Fig. 6A). In the control groups treated with either KLA or PBS, small amounts of necrotic regions were present. In contrast, the KLA-iRGD-treated group displayed larger necrotic regions, which explains the smaller volumes of the tumors in the group at the pathological level. Furthermore, tumors from the KLA-iRGD group showed significantly stronger TUNEL staining than tumors treated with PBS or KLA, indicating substantial cell death in the KLA-iRGD-treated tumors (Fig. 6B).

\section{Discussion}

As one of the main cause of cancer-related death worldwide, gastric cancer has a high rate of incidence and mortality (2). Accordingly, it is important to develop therapeutic agents with reduced side-effects and high specificity for gastric cancer (17). Several available cancer chemotherapy agents are limited by their serious side-effects and acquired resistance (18). Thus, there is an urgent need for a drug delivery system which delivers drugs at the target sites of tumor tissues. Phage display peptide libraries improve the therapeutic efficacy of 


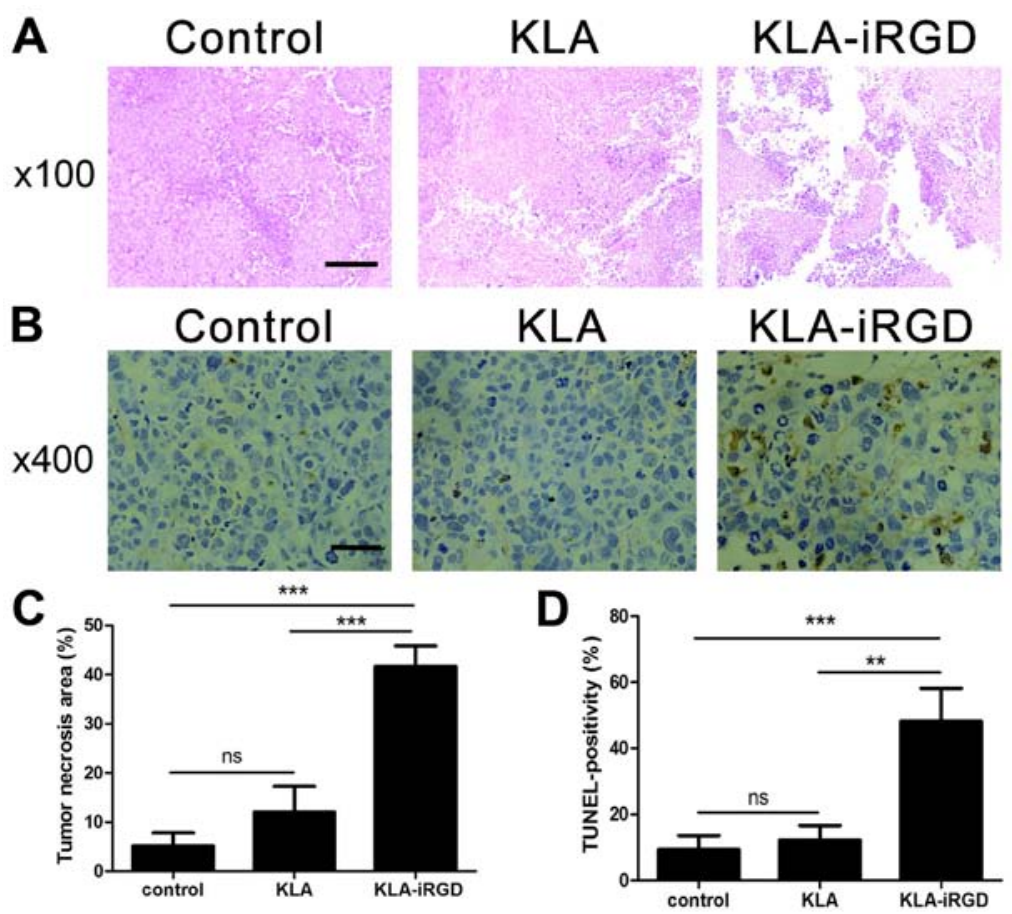

Figure 6. Effect of KLA-iRGD on the cell death of MKN45 tumors. (A) H\&E staining of tumor sections from mice treated with intraperitoneal injection of PBS, KLA or KLA-iRGD. Scale bar, $100 \mu \mathrm{m}$. (B) Cell death was evaluated by immunohistochemistry of TUNEL. Scale bar, $25 \mu \mathrm{m}$. (C and D) Quantification of (C) tumor necrosis area and (D) TUNEL immunohistochemical positivity. Error bars denote mean \pm SD; ${ }^{* *}$ p $<0.01,{ }^{* * *}$ p $<0.001$; ns, not significant.

anticancer drugs by providing peptides with high affinity and specificity, and in addition they typically have low interaction with the immune system and good tumor and tissue penetration (19). In the targeted delivery of anticancer drugs into cancer tissue the ability to penetrate deep into the solid tumor tissue can potentially be a great challenge for the targeted therapeutic (3). In solid cancer, the homeostatic regulation of tissue fails, cancer cells are in a hypoxic state, the pressure of interstitial fluid increases (20-23), and the extracellular matrix (ECM) impedes the motion of molecules and drugs into the tumor tissue (24-26).

As a pro-apoptotic peptide, KLA leads to cellular apoptosis by disrupting mitochondrial homeostasis and activating caspases. Caspase-activating cytochrome $c$ release from damaged mitochondria induces activation of caspases-9 and -3 , and mediates both early and late apoptosis $(17,27)$. Originally developed as an antibacterial peptide, KLA is non-toxic in eukaryotic cells as it cannot be actively internalized $(6,28)$. Indeed, since KLA fails to efficiently pass through eukaryotic plasma membranes (29), an alternative method has been developed that involves the integration of KLA with other drugs, which are more efficient than the individual therapy. In this regard, the pro-apoptotic activity of the targeted fusion protein KLA-iRGD was reported. The iRGD peptide was shown to follow a multistep tumor-targeting mechanism. The intact peptide binds to $\alpha v$ integrins expressed on the cell surface, where it is proteolytically cleaved to generate the fragment of CRGDK (15). Such fragmenst bind to neuropilin-1 and are internalized into tumor cells, thus penetrating tumor tissues (16). The cell-penetrating activity of iRGD is greater than that of common RGD peptides (15). It is actually greater than what can be accomplished with regular RGD peptides and mimics, which only deliver payloads to the tumor vessels.
In the present study, we coupled iRGD to KLA to study their antiproliferation and penetration activities on gastric cancer. We also showed that the KLA peptide is highly active when introduced into tumor cells using protein transduction mediated by the cell-internalizing peptide iRGD. Moreover, the present study showed that the fusion protein is an excellent antitumor agent which can be released and spread in tumor tissues through intravenous injection. In addition, in the present study, we demonstrated that these peptides provide a protein with anticancer activity with the ability to penetrate readily into tumor tissue. Furthermore, the in vitro cytotoxicity of KLA-iRGD to MKN45 cells was effectively enhanced. The results from the MTT assay indicated that KLA-iRGD clearly decreased cell viability. Flow cytometric analysis of cell apoptosis also demonstrated that KLA-iRGD increased the induction of both early and late apoptosis in the MKN45 cells. We also showed that KLA-iRGD is effective in suppressing the growth of gastric cancer cell lines in vivo. The improved antitumor activity of KLA-iRGD can be attributed to the iRGD peptide, which facilitates the internalization of the formulation into MKN45 cells.

A recombinant protein $\mathrm{iRGD}-\mathrm{CDD}$ was defined and constructed by Chen et al (30) who confirmed the extensive distribution of iRGD-CDD in tumors and showed that the iRGD peptide can be active in protein transduction. Similarly, in the present study, after intraperitoneal injection, the fusion protein KLA-iRGD was found to be a promising antitumor agent to spread in tumor tissue. We made use of the tumor penetration activity of KLA-iRGD in the development of a new anticancer treatment approach. The treatment of tumorbearing mice with KLA-iRGD effectively inhibited tumor growth in vivo, resulting in marked reduction in MKN45 tumor volume. Furthermore, KLA-iRGD did not cause detectable 
toxicity to important organs, such as the lung, liver, heart, spleen and kidney, in the tumor-bearing mice. KLA together with iRGD led to an improved pro-apoptotic effect, likely due to the high concentration of KLA in the targeted tumor cells.

To conclude, the present study provides an approach to construct a fusion protein of peptide KLA and iRGD to target tumors. The construct facilitated the entry of KLA into human MKN45 gastric carcinoma cells, where it decreased the growth of tumors in mice and exerted an apoptotic effect. There are still many issues associated with this approach that need to be addressed in order to ensure the enhanced clinical application, including pharmacokinetic properties and stability of the recombinant proteins, and the mechanisms underlying the antitumor effect of KLA-iRGD. We believe that KLA-iRGD could be utilized as an anticancer agent. However, further research is warranted to confirm this suggestion.

\section{Acknowledgements}

The present study was supported by the National Natural Science Foundation of China (grant nos. 81370064, 81502037 and 81572329).

\section{References}

1. Jemal A, Bray F, Center MM, Ferlay J, Ward E and Forman D Global cancer statistics. CA Cancer J Clin 61: 69-90, 2011.

2. Siegel R, Ma J, Zou Z and Jemal A: Cancer statistics, 2014. CA Cancer J Clin 64: 9-29, 2014

3. Sha H, Zou Z, Xin K, Bian X, Cai X, Lu W, Chen J, Chen G, Huang L, Blair AM, et al: Tumor-penetrating peptide fused EGFR single-domain antibody enhances cancer drug penetration into 3D multicellular spheroids and facilitates effective gastric cancer therapy. J Control Release 200: 188-200, 2015.

4. Refaat A, Abd-Rabou A and Reda A: TRAIL combinations: The new 'trail' for cancer therapy (Review). Oncol Lett 7: 1327-1332, 2014.

5. Foillard S, Jin ZH, Garanger E, Boturyn D, Favrot MC, Coll JL and Dumy P: Synthesis and biological characterisation of targeted pro-apoptotic peptide. Chembiochem 9: 2326-2332, 2008.

6. Ellerby HM, Arap W, Ellerby LM, Kain R, Andrusiak R, Rio GD, Krajewski S, Lombardo CR, Rao R, Ruoslahti E, et al: Anti-cancer activity of targeted pro-apoptotic peptides. Nat Med 5: 1032-1038, 1999.

7. Marks AJ, Cooper MS, Anderson RJ, Orchard KH, Hale G, North JM, Ganeshaguru K, Steele AJ, Mehta AB, Lowdell MW, et al: Selective apoptotic killing of malignant hemopoietic cells by antibody-targeted delivery of an amphipathic peptide. Cancer Res 65: 2373-2377, 2005.

8. Thornberry NA, Rosen A and Nicholson DW: Control of apoptosis by proteases. Adv Pharmacol 41: 155-177, 1997.

9. Thornberry NA and Lazebnik Y: Caspases: Enemies within. Science 281: 1312-1316, 1998.

10. Chu DS, Bocek MJ, Shi J, Ta A, Ngambenjawong C, Rostomily RC and Pun SH: Multivalent display of pendant pro-apoptotic peptides increases cytotoxic activity. J Control Release 205: $155-161,2015$.

11. Javadpour MM, Juban MM, Lo WC, Bishop SM, Alberty JB Cowell SM, Becker CL and McLaughlin ML: De novo antimicrobial peptides with low mammalian cell toxicity. J Med Chem 39: 3107-3113, 1996.

12. Hyun S, Lee S, Kim S, Jang S, Yu J and Lee Y: Apoptosis inducing, conformationally constrained, dimeric peptide analogs of KLA with submicromolar cell penetrating abilities. Biomacromolecules 15: 3746-3752, 2014.
13. Mai JC, Mi Z, Kim SH, Ng B and Robbins PD: A proapoptotic peptide for the treatment of solid tumors. Cancer Res 61: 7709-7712, 2001.

14. Ma JL, Wang H, Wang YL, Luo YH and Liu CB: Enhanced Peptide delivery into cells by using the synergistic effects of a cell-penetrating Peptide and a chemical drug to alter cell permeability. Mol Pharm 12: 2040-2048, 2015.

15. Sugahara KN, Teesalu T, Karmali PP, Kotamraju VR, Agemy L, Girard OM, Hanahan D, Mattrey RF and Ruoslahti E: Tissue-penetrating delivery of compounds and nanoparticles into tumors. Cancer Cell 16: 510-520, 2009.

16. Sugahara KN, Teesalu T, Karmali PP, Kotamraju VR, Agemy L, Greenwald DR and Ruoslahti E: Coadministration of a tumorpenetrating peptide enhances the efficacy of cancer drugs. Science 328: 1031-1035, 2010.

17. Fu B, Long W, Zhang Y, Zhang A, Miao F, Shen Y, Pan N, Gan G, Nie F, He Y, et al: Enhanced antitumor effects of the BRBP1 compound peptide BRBP1-TAT-KLA on human brain metastatic breast cancer. Sci Rep 5: 8029, 2015.

18. Bryde S and de Kroon AI: Nanocapsules of platinum anticancer drugs: Development towards therapeutic use. Future Med Chem 1: 1467-1480, 2009.

19. Wang Y, Xiao W, Zhang Y, Meza L, Tseng H, Takada Y, Ames JB and Lam KS: Optimization of RGD-containing cyclic peptides against $\alpha v \beta 3$ integrin. Mol Cancer Ther 15: 232-240, 2016.

20. Jain RK: The Eugene M. Landis Award Lecture 1996. Delivery of molecular and cellular medicine to solid tumors. Microcirculation 4: 1-23, 1997.

21. Milosevic MF, Fyles AW, Wong R, Pintilie M, Kavanagh MC, Levin W, Manchul LA, Keane TJ and Hill RP: Interstitial fluid pressure in cervical carcinoma: Within tumor heterogeneity, and relation to oxygen tension. Cancer 82: 2418-2426, 1998.

22. Heldin CH, Rubin K, Pietras K and Ostman A: High interstitial fluid pressure - an obstacle in cancer therapy. Nat Rev Cancer 4: 806-813, 2004.

23. Li R, Xie L, Zhu Z, Liu Q, Hu Y, Jiang X, Yu L, Qian X, Guo W, Ding Y, et al: Reversion of $\mathrm{pH}$-induced physiological drug resistance: A novel function of copolymeric nanoparticles. PLoS One 6: e24172, 2011.

24. Netti PA, Berk DA, Swartz MA, Grodzinsky AJ and Jain RK: Role of extracellular matrix assembly in interstitial transport in solid tumors. Cancer Res 60: 2497-2503, 2000.

25. Davies CL, Berk DA, Pluen A and Jain RK: Comparison of IgG diffusion and extracellular matrix composition in rhabdomyosarcomas grown in mice versus in vitro as spheroids reveals the role of host stromal cells. Br J Cancer 86: 1639-1644, 2002.

26. Brown E, McKee T, diTomaso E, Pluen A, Seed B, Boucher Y and Jain RK: Dynamic imaging of collagen and its modulation in tumors in vivo using second-harmonic generation. Nat Med 9: 796-800, 2003.

27. Ma X, Jia J, Cao R, Wang X and Fei H: Histidine-iridium (III) coordination-based peptide luminogenic cyclization and cyclo-RGD peptides for cancer-cell targeting. J Am Chem Soc 136: 17734-17737, 2014.

28. Agemy L, Friedmann-Morvinski D, Kotamraju VR, Roth L, Sugahara KN, Girard OM, Mattrey RF, Verma IM and Ruoslahti E: Targeted nanoparticle enhanced proapoptotic peptide as potential therapy for glioblastoma. Proc Natl Acad Sci USA 108: 17450-17455, 2011.

29. Alves ID, Carré M, Montero MP, Castano S, Lecomte S, Marquant R, Lecorché P, Burlina F, Schatz C, Sagan S, et al: A proapoptotic peptide conjugated to penetratin selectively inhibits tumor cell growth. Biochim Biophys Acta 1838: 2087-2098, 2014.

30. Chen R, Braun GB, Luo X, Sugahara KN, Teesalu T and Ruoslahti E: Application of a proapoptotic peptide to intratumorally spreading cancer therapy. Cancer Res 73: 1352-1361, 2013. 